\title{
VALORACIÓN DE EMPRESAS POR DESCUENTO DE FLUJOS DE CAJA: PROYECCIÓN DE RATIOS Y ESTIMACIÓN DEL VALOR TERMINAL POR MÚLTIPLOS
}

\author{
Luis Blanco Pascual, Universidad de La Rioja \\ Luis González Jiménez, Universidad de La Rioja \\ Juan Carlos Ayala Calvo, Universidad de La Rioja
}

\section{RESUMEN}

El modelo del descuento de flujos de caja está considerado como uno de los modelos estándar de valoración de activos. Además de la selección y estimación de las tasas de descuento, el principal problema para su aplicación en la práctica es el modelado de la corriente de flujos a descontar. Cuando el activo valorado es una empresa este problema es especialmente relevante, ya que normalmente se asume que las empresas tienen un duración indeterminada en el tiempo. Por tanto, teóricamente el horizonte de valoración tiende a infinito, no existe valor terminal para la inversión, y debe estimarse una corriente ilimitada de flujos de caja. La solución básica que suele adoptarse consiste en realizar una proyección limitada de los flujos a descontar, y estimar el valor terminal (o valor de salida) mediante una perpetuidad.

El presente trabajo extiende lo anterior de la siguiente manera: (i) la corriente de flujos a descontar se proyecta a partir de las ventas, el ratio flujo de caja a ventas, y sus tasas de variación, y (ii) se estudian distintas alternativas para la estimación y cálculo del valor terminal mediante múltiplos de valoración relativa.

El modelo se aplica a una muestra de empresas del índice Standard \& Poor’s 500.

PALABRAS CLAVE: valoración de empresas, ratios de ventas, valoración relativa, valor terminal.

\section{ABSTRACT}

The discounted cash-flow model is well established as the standard tool for the valuation of assets. Choice of the adequate discount rates aside, the modeling of the expected cash-flow stream is the main problem for application in practice. Where company valuation is concerned, this is specially true because the asset is assumed to last forever or, at least, for as long as it actually matters. Thus, theoretically the time horizon tends to infinity, there is no terminal value and an unlimited series of cash-flows would need to be forecasted. The staple solution is well known: a cash-flow stream forecast limited to a few years and a terminal value computed as a perpetuity. This possibility notwithstanding, this paper explores other options for estimating the last expected cash-flow (i.e., the terminal or exit value) using two Enterprise multiples: sales and EBITDA multiples. In addition, it addresses alternative ways to project the cash-flow stream up to the investor's exit point using sales ratios and their rates of change, and the expected sales growth.

The model is illustrated with its application to a sample of firms in the Standard \& Poor's 500 index.

KEY WORDS: firm valuation, sales’ ratios, relative valuation, exit value. 


\section{1.- INTRODUCCIÓN}

Uno de los temas principales dentro de la investigación en finanzas es la valoración de activos de distinta naturaleza, especialmente la valoración de empresas (bien de su valor total, es decir incluida la deuda financiera, bien de la porción perteneciente en exclusiva a sus accionistas). Por múltiples motivos, el proceso de valoración es fundamental. Decidir el atractivo de un nuevo negocio, afrontar con éxito operaciones de compraventa de empresas, negociar nueva financiación u operar en el mercado bursátil requieren estimar el valor de la empresa en estudio. Además, es imprescindible para evaluar la calidad de la gestión y perseguir el objetivo de crear valor para el accionista, que depende de la progresión del valor de la empresa.

Los problemas surgen debido a que no existe un único enfoque para el tratamiento del problema. A lo largo de la historia, ha sido constante tanto la búsqueda de nuevos modelos de valoración, como la mejora de los modelos ya existentes. Además, no se ha establecido una clara distinción entre lo que es un modelo de valoración, una variante de un modelo, y método de valoración. Por tanto, es necesario en primer lugar distinguir los siguientes términos: modelos, variantes o desarrollos de un modelo, y métodos.

Un modelo es una herramienta matemática, compuesta por una o varias ecuaciones analíticas, que representa de forma general situaciones complejas. Variantes o desarrollos del modelo, son variaciones efectuadas para incorporar las características específicas del activo valorado, y la información disponible en el modelo general. Un método, proporciona una guía de actuación a la hora de recoger y manipular los datos necesarios para alimentar el modelo, para la interpretación de resultados, y para el establecimiento de reglas de decisión basadas en estos últimos.

De acuerdo con lo anterior, según Damodaran (2001) existen en finanzas dos modelos generales de valoración: el Modelo del Descuento de Flujos de Caja ( Discounted Cash Flow Model, en adelante DCFM) y el Modelo de Opciones Reales (MOR).

El MOR, surge ante la imposibilidad de valorar mediante el DCFM tradicional, cualquier tipo de activo, empresa o proyecto, que incorpore algún tipo de flexibilidad futura. Una opción real está presente en un proyecto de inversión cuando existe una posibilidad futura de actuación, una vez que se ha despejado la incertidumbre inicial acerca de la evolución de un determinado acontecimiento. No obstante, el MOR ha tenido escasa aceptación en el ámbito profesional, debido seguramente a la enorme cantidad de datos necesaria para alimentar el modelo y a su extremada complejidad (Trahan y Gitman, 1995; Graham y Harvey, 2001).

El DCFM determina el valor de cualquier activo, como el valor descontado de una serie de flujos de dinero previstos que dicho activo generará en el futuro, descontados a una tasa apropiada, en función del riesgo asociado a dichos flujos. Normalmente suele realizarse una proyección detallada de los flujos de dinero generados durante un período de tiempo denominado horizonte de la inversión u horizonte temporal. Al final del mismo, se determina el valor terminal de la inversión, que es descontado también hasta el momento inicial, como si de un flujo más se tratase. Es el método de valoración más extendido en la práctica profesional, y al que más páginas se le han dedicado en la literatura académica.

Las variantes y métodos derivadas del DCFM son numerosos, y la búsqueda de nuevas versiones es continua. Entre sus variantes destacan los Métodos de Valoración Relativa (MVR), o Método de Múltiplos de Valoración, que estiman el valor de un activo basándose en cómo valora el mercado activos de similar 
naturaleza. Los MVR, especialmente en los últimos años, se han consolidado como el método de valoración de empresas más utilizado en la práctica (Damodaran, 2002), puesto que necesitan del establecimiento de bastantes menos hipótesis que el DCFM tradicional, y porque es más probable que las estimaciones obtenidas por estos métodos, reflejen la tendencia actual del mercado, ya que el objetivo perseguido es la obtención de una medida relativa del activo valorado, y no la obtención del valor intrínseco del mismo. Autores como Baker y Ruback (1999), y Kaplan y Ruback $(1995,1996)$ señalan incluso que la combinación del DCFM junto con los múltiplos de valoración permite obtener resultados más satisfactorios en la valoración de empresas que la aplicación aislada del modelo de descuento de flujos. Este es precisamente el punto de partida del modelo discutido en el presente trabajo.

El objetivo de este trabajo es el desarrollo, y posterior contrastación empírica, de una variante del modelo de descuento de flujos de caja en dos etapas. En la primera de ellas, de duración limitada en el tiempo ( $n$ períodos), la corriente de flujos de caja a descontar se proyecta en función de: las ventas, la relación lineal (ratio) entre flujos de caja y ventas (CF/S), y finalmente en función de las tasas de variación de ambas variables. En la segunda se utilizan dos múltiplos de valoración relativa para estimar el valor terminal de la inversión: el múltiplo Valor Total de la Empresa-Ventas (EV/S), y el múltiplo Valor Total de la Empresa-EBITDA ${ }^{1}$ (EV/E). De esta manera, se pretende comprobar la eficiencia en términos valorativos de la combinación del DCFM con dos de los múltiplos del valor total de la empresa más utilizados en la práctica.

\section{2.- MODELO DE VALORACIÓN DE EMPRESAS POR DESCUENTO DE FLUJOS DE CAJA: EXPRESIÓN GENERAL Y VARIANTES}

El valor de cualquier activo (Ecuación 1) puede expresarse en función de los flujos de caja esperados durante un determinado período de tiempo, denominado horizonte temporal, descontados a una tasa que mide el riesgo asociado a dichos flujos. De esta manera, el valor de cualquier empresa puede determinarse como el valor actual de una serie de flujos futuros. Si los flujos descontados son los disponibles (teóricamente) tanto para los accionistas como para los proveedores de deuda (flujos de caja libres) obtenemos una estimación del valor de la empresa en el momento actual.

$$
V_{0}=\sum_{i=1}^{n} Q_{i} \prod_{h=1}^{i}\left(1+r_{h}\right)^{-1}
$$

Mediante la expresión anterior obtenemos el valor del activo $\left(\mathrm{V}_{0}\right)$ en función de la tasa de descuento apropiada $\left(r_{h}\right)$ y los flujos de caja netos $\left(Q_{i}\right)$ que se espera obtener durante los $n$ períodos de tiempo comprendidos entre el momento inicial y final del horizonte de inversión.

Para obtener el valor total de la empresa, es decir incluyendo el valor del patrimonio neto y de la deuda financiera, $r_{h}$ debe ser el coste medio ponderado (después de impuestos) de todas las fuentes de financiación, y $Q_{i}$ el flujo de caja libre disponible tanto para los accionistas como para los poseedores de deuda (o flujo neto de caja libre, Free Cash Flow to Enterprise, en adelante FCFE).

\footnotetext{
${ }^{1}$ Earnings before interests, taxes, depreciation and amortization, es decir beneficio operativo antes de intereses, impuestos, y amortización del inmovilizado y fondo de comercio.
} 
La mayoría de las adaptaciones de este modelo para la valoración de empresas (Ecuación 1) asumen que el horizonte temporal tiende a infinito. Algunas variantes (Damodaran, 1994; Damodaran, 2002) dividen el horizonte temporal en dos o en tres intervalos, cada uno de los cuáles representa comportamientos distintos de los FCFE a través de tasas de crecimiento de dichos flujos distintas en cada intervalo. De la misma manera, la mayoría de las variantes del modelo, bien para la valoración del patrimonio neto, es decir de la parte de la empresa perteneciente a sus accionistas (Gordon y Shapiro, 1956; Gordon, 1962; Ohlson, 1995), bien para la valoración de la empresa en su totalidad (Koller, Goedhart y Wessels, 2005; Kruschwitz, 2006), de manera explícita (Gordon y Shapiro, 1956; Gordon, 1962) o implícita (Ohlson, 1995), asumen que los FCFE crecen a una tasa constante. Igualmente, salvo excepciones (Koller, Goedhart y Wessels, 2005; Kruschwitz, 2006), se asume que la tasa de descuento permanece constante durante todo el horizonte de valoración $\left(r_{h}=k: h=\right.$ $1, \ldots, n)$.

En cuanto a la formulación del modelo, aquí se utiliza la siguiente ecuación (equivalente a la Ecuación 1), más manejable desde un punto de vista matemático:

$$
V_{0}=\sum_{i=1}^{n} Q_{i} e^{-\sum_{h=1}^{i} k_{h}}
$$

Donde $k_{h}=\ln \left(1+r_{h}\right)$. De la misma manera las tasas de variación se expresarán en base logarítmica.

Una variante muy utilizada en la literatura académica del DCFM (Damodaran, 2002; Ohlson y Zhang, 1999), trabaja con las siguientes simplificaciones, dividiendo el horizonte de valoración en dos intervalos:

1) Un primer intervalo de duración $n$ períodos (entre cinco y diez años), donde se realiza una proyección detallada de los flujos de caja. Normalmente dichos flujos son estimados de manera individual, sin necesidad de establecer una relación funcional entre ellos.

2) Para el segundo intervalo de duración $(n+1, \infty)$, se calcula un valor terminal o valor de salida mediante una renta perpetua, aplicando una tasa de crecimiento constante al último flujo de caja estimado en el primer intervalo $Q_{n}$.

Partiendo de la Ecuación 2, el resultado sería el siguiente:

$$
V_{0}=\sum_{i=1}^{n} Q_{i} e^{-i k}+Q_{n} e^{-n k+g} \frac{1}{1-e^{g-k}}
$$

Donde $k$ es la tasa de descuento constante durante todo el horizonte de valoración, y $g$ es la tasa de crecimiento constante a perpetuidad. Es importante señalar, que en la estimación de la tasa de descuento $k$ hay que tener en cuenta si el valor que se está calculando corresponde al valor total de la empresa (es decir, incluyendo el valor de la deuda financiera), o el valor del patrimonio neto (es decir, de la porción perteneciente únicamente a sus accionistas). En el primer caso, $k$ debe ser el coste medio ponderado (después de impuestos) de todas las fuentes de financiación de la empresa; en el segundo caso, cuando los flujos de caja se presentan netos del servicio de la deuda, $k$ debe ser el coste de los recursos propios $\left(k_{e}\right)$ calculado normalmente mediante el CAPM (Capital Asset Pricing Model). La Ecuación 4 representa la expresión general del CAPM: 


$$
k_{e}=r_{f}+\beta\left(E\left[R_{m}\right]-r_{f}\right)
$$

La tasa de rentabilidad libre de riesgo $\left(r_{f}\right)$ suele estimarse normalmente a partir de la curva de tipos de interés (zero coupon yield curve rate), en el vencimiento coincidente con el final del horizonte de valoración. Respecto al coeficiente $\beta$ y a la esperanza de la rentabilidad de mercado $\left(E\left[R_{m}\right]\right)$ la clave reside en la elección del índice bursátil de referencia (el índice de mercado donde la empresa cotiza, o el correspondiente índice sectorial). En el caso de que la valoración se esté efectuando para una empresa no cotizada, según Damodaran $(2001,2002)$ las dos variables anteriores deben estimarse a partir de empresas cotizadas comparables.

Autores como Damodaran $(1984,2001,2002)$ proponen, en función de la empresa objeto de valoración, incluir un intervalo más de valoración, de duración $p$ períodos, que represente por ejemplo una etapa de desaceleración en el crecimiento de una empresa. De esta manera, dicho intervalo intermedio, sirve de enlace entre una etapa inicial de fuerte crecimiento (y duración $n$ períodos), y el estado estacionario (de crecimiento constante a perpetuidad, $g$ ) alcanzado en $n+p$. La ecuación resultante es la siguiente:

$$
V_{0}=\sum_{i=1}^{n} Q_{i} e^{-i k}+e^{-n k} \sum_{i=1}^{p} Q_{n} e^{i(g-k)}+e^{-(n+p) k} \frac{Q_{n} e^{p g+g^{\prime}}}{1-e^{g^{\prime}-k}}
$$

\section{3.- PARÁMETROS TEMPORALES Y VALOR TERMINAL}

La literatura académica ha dedicado considerables esfuerzos a la investigación de cómo afecta el horizonte temporal a la valoración de activos (Penman, 1998a; Ohlson y Zhang, 1999). Esencialmente, dichos autores concluyen que el error cometido en la valoración, disminuye de manera monótona conforme aumenta el horizonte temporal. Sin embargo, la práctica impone ciertas restricciones que han de ser consideradas, dada la especial relevancia que tiene en el tema que nos ocupa. Por ejemplo, respecto al primero de los intervalos de valoración, en el cuál se realiza una proyección detallada de los flujos de caja, Ohlson y Zhang (1999) afirman que no puede extenderse más de un determinado número de períodos (hasta quince años). El motivo es la inviabilidad de poder realizar estimaciones fiables más allá de ese momento sobre la evolución, bien del flujo de caja, bien de las variables participantes en su cálculo ( ventas, variación del circulante, etc.).

No obstante, es posible encontrar cierta racionalidad en la elección de los parámetros temporales $n$ y $p$ de la Ecuación 5. En este sentido, Dehning et al.(2005) proporcionan una interesante visión de los factores determinantes del resultado empresarial. De acuerdo con ellos, los rendimientos empresariales se deben principalmente a factores específicos de la empresa en cuestión y, de manera secundaria a factores específicos del sector de actividad de pertenencia, resultando en lo que ellos definen como industry abnormal earnings (IAE) y firm abnormal earnings (FAE). En una economía de libre mercado parece razonable asumir que las ventajas competitivas de una empresa respecto del resto de las pertenecientes al sector desaparecen en un determinado momento del tiempo y con ellas los FAE. De la misma manera, sectores con crecimientos superiores a la economía acaban por alinearse con esta, desapareciendo entonces los IAE. En consecuencia, la estimación de los parámetros temporales puede basarse en las expectativas de duración e intensidad de las ventajas competitivas derivadas de los FAE e IAE, y utilizar como tasa de crecimiento de las ventas a largo 
plazo la tendencia del Producto Interior Bruto (PIB) en términos nominales. Aunque esto último ya ha sido sugerido por algunos autores (Damodaran, 1994), actualmente los profesionales del análisis financiero tienden a utilizar tasas de crecimiento a largo plazo que equivalen a tasas de crecimiento negativas en términos reales. Dados los tipos de interés tan bajos que actualmente prevalecen en el mercado, esta parece ser la única solución posible para no obtener valoraciones fuera de rango (cuando se comparan con valores de mercado).

\section{1.- Valor terminal: alternativas de cálculo}

Existen básicamente tres alternativas para estimar el valor terminal o de salida (exit value en adelante $\mathrm{XV})$ :

1) XV calculado como una perpetuidad (renta perpetua de crecimiento constante).

2) XV calculado mediante múltiplos de valoración relativa ( múltiplos de ventas, múltiplo del EBITDA, etc.).

3) XV calculado como valor de liquidación del activo.

En la primera de las alternativas anteriores suele utilizarse (Damodaran, 1994) como tasa de crecimiento constante la ya mencionada tendencia del Producto Interior Bruto (PIB). Esta hipótesis parece razonable siempre que no se espere obtener resultados anormales (tanto para la empresa como para el sector) a partir de un determinado momento en el tiempo (coincidente con el punto de salida, o punto en el que el valor terminal se calcula por cualquiera de las anteriores alternativas).

La segunda alternativa consiste en calcular el XV mediante la utilización de múltiplos de valoración relativa. En la última década, los Métodos de Valoración Relativa (MVR) se han convertido en el método de valoración de empresas más extendido en la práctica profesional. Lo que en cierta manera es consistente con la propuesta de combinar múltiplos de valoración con el DCFM (Baker y Ruback, 1999; Kaplan y Ruback, 1995, 1996). Las valoraciones obtenidas parecen estar más próximas a las valoraciones efectuadas por el mercado, permiten ampliar el rango de valoraciones de un mismo activo y, dependiendo de las características de este último y del uso que se vaya a hacer de la valoración (la salida de una inversión mediante OPV, o mediante una colocación privada de acciones) elegir el más adecuado.

Por tanto, los MVR proporcionan una alternativa, o tantas como diferentes múltiplos puedan ser aplicados de forma razonable, para calcular el valor terminal. El procedimiento de aplicación es sencillo:

$$
X V=m_{I} \times v_{t}
$$

Donde $m_{I}$ es el múltiplo representativo del grupo de empresas comparables a la empresa objeto de valoración (múltiplo del neto o del valor total de la empresa, en función del valor que se desee obtener), y $X V$ es el valor estimado que toma el conductor de valor ${ }^{2}$ de la empresa valorada en el momento $t$ del tiempo (punto de salida y cálculo del valor terminal).

El procedimiento anterior puede ser objeto de mejora si se ajusta el primero de los dos factores (múltiplo del grupo comparable) de manera que refleje la posición esperada de la empresa respecto a su sector en

\footnotetext{
${ }^{2}$ El indicador o conductor de valor, es una magnitud vinculada con el valor de la empresa, seleccionada por el usuario, de entre aquellas más claramente relacionadas con los rendimientos futuros y con la creación de valor ( ventas, EBITDA, beneficio neto, etc.).
} 
el punto de salida. De esta manera, puede resolverse en parte la principal debilidad de los Métodos de Valoración Relativa: el hecho de que no se tengan en cuenta las diferencias, que en términos de eficiencia o potencial de crecimiento futuro, existan entre la empresa valorada y el grupo de comparación.

Aunque muchos autores sostienen que, en función del sector de actividad, ciertos múltiplos de valoración permiten obtener mejores resultados que otros (Tasker, 1998; Baker y Ruback, 1999), la opinión más generalizada y comúnmente aceptada es que la validez valorativa de los MVR reside en una definición consistente (desde el punto de vista analítico) del múltiplo de valoración (Alford, 1992; Beatty et al., 1999; Bhojraj y Lee, 2002; Damodaran, 2001; Eberhart, 2001; Kim y Ritter, 1999; Liu et al., 2002) y en el criterio de selección del grupo de empresas comparables. Respecto a este último, el criterio de selección de comparables más utilizado es el sector de pertenencia, asumiendo que las empresas del mismo sector presentan características muy similares en cuanto a niveles de riesgo, generación de flujos de caja y potencial de crecimiento futuro.

Por último, la tercera alternativa estima el valor terminal mediante el valor de liquidación, entendido como el valor esperado de los activos netos de la empresa en el punto de salida. Esta alternativa únicamente tiene sentido si el punto de salida de la inversión está muy distante en el tiempo, una vez que los activos intangibles de la empresa se encuentren totalmente consumidos y ya no es posible la generación de resultados anormales, tanto los derivados de la propia empresa como los derivados del sector (FAE e IAE).

Aunque las tres alternativas anteriores no son mutuamente excluyentes, por cuanto permiten ampliar el rango de resultados de valoración, el presente trabajo únicamente se ocupa de la segunda de ellas.

\section{4.- PROYECCIÓN DE LOS FLUJOS DE CAJA EN FUNCIÓN DE LAS VENTAS, EL RATIO FLUJO DE CAJA-VENTAS, Y SUS TASAS DE VARIACIÓN}

En esta sección se desarrolla una variante del DCFM basada en la proyección de ratios contables. En buena medida cabe afirmar que dicha variante se apoya en el Análisis Fundamental por su capacidad para predecir magnitudes asociadas con la actividad de la empresa ${ }^{3}$, capacidad que motiva la especial relevancia cobrada en los últimos años por el Análisis Fundamental aplicado a la investigación en los mercados de capitales $^{4}$ (Giner, 2001; Giner et al., 2002; Kothari, 2001).

Aunque la práctica profesional parece ser consistente con la variante que aquí se propone, la literatura académica sobre la utilización de ratios contables para la proyección de flujos de caja es escasa. Como excepción a lo anterior, destaca el trabajo de Nissim y Penman (2001). Dichos autores diseñan un procedimiento de análisis de estados financieros mediante ratios, que permite la predicción y valoración del neto patrimonial de una muestra de empresas.

El diseño del modelo de valoración que aquí se estudia consta de dos partes (o intervalos de valoración):

1) En la primera se realiza un proyección detallada de los flujos de caja durante los $n$ primeros períodos, a partir de: (i) una variable financiera fundamental (ventas, EBITDA, beneficio neto,

\footnotetext{
3 Entre otros, Beaver y Morse (1978), Beaver y Ryan (2000), Cragg y Malkiel (1982), Fama y French (2000), Kothari (2001), Molodovsky (1953), Peasnell (1982), Penman (1996,1998b), Preinreich (1938), y Ryan (1995).

${ }^{4}$ Capital markets research in accounting, o Market based accounting research.
} 
etc.), (ii) la relación lineal (mediante ratio) entre flujos de caja y dicha variable, y (iii) las tasas de variación de ambas variables.

2) En la segunda se estima el valor terminal de la inversión por múltiplos de valoración que puedan o no utilizar en su cálculo como conductor de valor la variable financiera fundamental definida en el punto anterior ${ }^{5}$.

\section{1.- Primer intervalo: modelado analítico de los flujos de caja}

Cuando se realiza una proyección detallada de los flujos de caja, más que la estimación del flujo en sí mismo, lo habitual es proyectar sus componentes de cálculo. La combinación más utilizada se muestra en la Figura 1:

\begin{tabular}{|c|}
\hline + EBITDA \\
\hline - Inversión en capital \\
\hline \pm Variación del Capital Circulante \\
\hline - Impuestos \\
\hline$=$ FCFE \\
\hline Donde Impuestos= (EBITDA-Amortización) $\times$ t \\
\hline
\end{tabular}

Figura 1: Flujo de caja libre.

Por tanto, el flujo de caja libre, $Q_{i}$ de la Ecuación 3 puede expresarse como la suma de sus $m$ determinantes o componentes:

$$
Q_{i}=\sum_{j=1}^{m} q_{j, i}
$$

El valor de cada una de esas $m$ componentes puede estimarse de manera individual para cada uno de los períodos del intervalo, o alternativamente puede calcularse en función del valor que toma en el primer período y sus posteriores tasas de variación, es decir:

$$
q_{j, i}=q_{j, 1} \cdot e^{\sum_{k=2}^{i} g_{j, k}}
$$

Supongamos que el crecimiento o variación de cada una de esas componentes es constante $\left(g_{j}\right)$ durante los $n$ períodos de duración del primer intervalo: $g_{j, k}: k=2, \ldots n$

Supongamos también, que existe una relación lineal significativa entre cada una de esas $m$ componentes y una determinada magnitud financiera. Si por ejemplo dicha magnitud fuese las ventas, y la relación lineal fuese mediante cociente o ratio ( margen operativo, margen neto, etc.), entonces $q_{j, i}$ puede reformularse como:

$$
q_{j, i}=r_{j, i} \cdot S_{i}
$$

\footnotetext{
${ }^{5}$ Lo relevante en este caso es que si se está estimando el valor total de la empresa, el múltiplo utilizado debe ser un múltiplo del valor total (incluida deuda) de la misma. No obstante parece razonable, que el múltiplo utilizado utilice como conductor de valor la variable financiera empleada para proyectar los flujos en el primer intervalo.
} 
Donde $r_{j, i}: j=1, \ldots, m$ son los ratios asociados a cada una de las $m$ componentes de $Q_{i}$, y $s_{i}$ las ventas del período i-ésimo. Si tenemos en cuenta que las tasas de variación de cada componente de cálculo de flujo son constantes para todo el primer intervalo, los valores del ratio y de las ventas en cada uno de los períodos posteriores al primero $(i>1)$ pueden ser expresados en función del valor que toman en el primer período y en función de sus tasas de variación en el intervalo $(2, i)$ :

$$
r_{j, i} \cdot S_{i}=r_{j, 1} \cdot e^{(i-1) h_{j}} S_{1} \cdot e^{(i-1) h_{s}}
$$

Con $j$ variando desde 1 hasta $m$, y representando $h_{j}$ y $h_{s}$ las tasas de variación del ratio y de las ventas respectivamente.

Mediante la aplicación del procedimiento anterior se obtiene la corriente de flujos del primer intervalo. No obstante, con el objetivo de simplificar la expresión analítica, el modelo que se aplica en este trabajo no se descompone el flujo de caja libre en sus componentes de calculo, sino que utiliza para la proyección en el primer intervalo el ratio flujo de caja-ventas. En futuros trabajos se pretende extender lo anterior, es decir establecer los ratios entre las distintas componentes de cálculo y las ventas, para poder contrastar si mejoran los resultados de la valoración (o por el contrario es indiferente), al proyectar de forma agregada el ratio flujo de caja-ventas como en este caso, o de manera individual para cada una de sus componentes.

Por tanto, la expresión que permite obtener el valor descontado de los flujos de caja previstos en el primer intervalo $\left(V_{1}\right)$ es la siguiente:

$$
V_{1}=r_{1} s_{1} e^{-k} \frac{1-e^{\left(g_{r}+g_{s}-k\right) n}}{1-e^{g_{r}+g_{s}-k}}
$$

Donde $r_{1}$ representa el valor que toma el ratio flujo de caja-ventas en el primer período (y $g_{r}$ su tasa de variación), $s_{1}$ es la cifra de ventas en el primer período (y $g_{s}$ su tasa de variación), y $k$ la tasa de descuento (coste medio ponderado de todas las fuentes de financiación).

\section{2.- Segundo intervalo: valor terminal por múltiplos}

Esta alternativa se aplica con dos de los múltiplos del valor de la empresa más utilizados en la práctica (Damodaran, 2002), el múltiplo Valor Total de la Empresa-Ventas (EV/S), que relaciona mediante cociente, el valor total de la empresa (incluida la deuda) con las ventas, y el múltiplo Valor Total de la Empresa-EBITDA (EV/E), que relaciona mediante cociente, el valor total de la empresa con el EBITDA. Normalmente, el valor de la empresa se obtiene como suma del valor de mercado de las acciones, más el valor contable de la deuda (Kaplan y Ruback, 1995; Damodaran, 2002).

\section{Múltiplo EV/S y EV/E}

El valor terminal de la inversión utilizando esta alternativa se obtiene de la siguiente manera:

$$
V_{2}^{\prime}=e^{-n k} x_{1} e^{(n-1)\left(g_{x}\right)} m_{s}
$$

Donde $m_{s}$ es, respectivamente, el múltiplo EV/S o el múltiplo EV/E histórico medio de la empresa valorada, $x_{1}$ el valor que toma el conductor de valor (ventas o EBITDA) en el primer período del horizonte de 
valoración, y $g_{x}$ la tasa de variación que dicho conductor de valor experimenta en cada uno de los $n$ primeros períodos. Por tanto:

$$
V_{0}=V_{1}+V_{2}^{\prime}=r_{1} s_{1} e^{-k} \frac{1-e^{\left(g_{r}+g_{s}-k\right) n}}{1-e^{g_{r}+g_{s}-k}}+e^{-n k} x_{1} e^{(n-1)\left(g_{x}\right)} m_{s}
$$

\section{5.- APLICACIÓN DEL MODELO DE VALORACIÓN: ESTUDIO EMPÍRICO}

El modelo de valoración se ha contrastado en una muestra de empresas del índice bursátil S\&P500, pertenecientes a su vez a la División D "Manufacturing" de la clasificación de sectores de actividad SIC (Standard Industrial Classification ${ }^{6}$ ). Se ha elegido la división de actividad anterior ya que es la que cuenta con mayor representatividad en el índice S\&P500, con un total de 114 empresas. De las empresas pertenecientes a la muestra inicial (114 empresas), únicamente han sido seleccionadas 64 empresas (57\% del total) que cumplen los dos requisitos siguientes:

1) Que para todos los trimestres comprendidos entre los ejercicios 2000-2005 (24 trimestres en total) existan datos disponibles para todas las variables del modelo.

2) Que el flujo de caja calculado tal y como se ha descrito anteriormente, sea positivo para cada empresa y período trimestral.

\footnotetext{
${ }^{6}$ La “Standard Industrial Classification” (SIC) es un sistema de clasificación de sectores de actividad económica (cuatro dígitos) propuesto por el gobierno americano, y establecido por vez primera en 1931. Dicha clasificación está siendo sustituida desde 1997, por la “North American Industry Classification System-NAICS” (seis dígitos). Sin embargo, ciertos departamento o agencias, como por ejemplo la U.S. Securities and Exchange Commission (SEC), utilizan todavía la clasificación SIC.
} 


\section{Variables utilizadas y fuentes de datos}

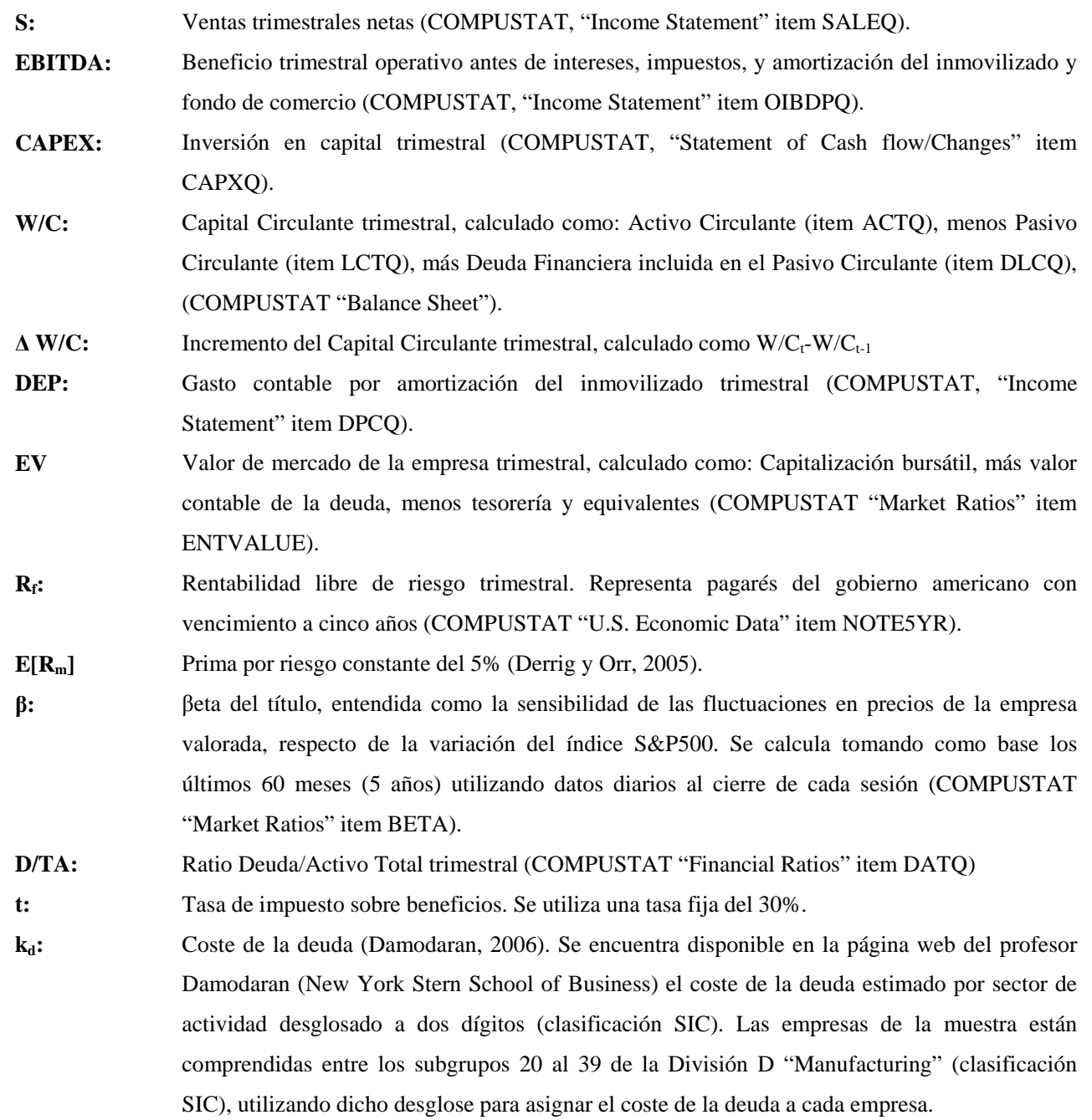

\section{Período de estudio, división y número de observaciones}

El horizonte de estudio abarca los ejercicios 2000-2005. Para ampliar el número total de observaciones, el modelo se aplica a cada empresa en cada trimestre. Por tanto es necesario disponer de datos trimestrales para cada variable, lo cuál es posible, gracias a que la base de datos utilizada (COMPUSTAT "North America") facilita datos segmentados por trimestres.

No obstante, y con el objetivo de evitar la estacionalidad que alguna de las variables (ventas, inversión en capital, etc.) pueda presentar, cada observación (trimestral), comprende un período de 12 meses consecutivos (twelve trailing month), que finalizan el último día del trimestre en el cual se realiza la valoración. Es decir, si por ejemplo nos encontramos en el 3T04 (tercer trimestre del 2004), el período valorado comprende el último trimestre del 2003 (4T2003) y los tres primeros del 2004 (123T2004). De esta manera, cuando la variable utilizada en el cálculo es una variable flujo (ventas o inversión en capital), el valor que se da a la variable es la suma de sus últimos cuatro valores trimestrales. Por contra, si es una variable fondo (Activo Total, Deuda Financiera, etc.) el valor que se da a la variable es el promedio de sus últimos cuatro valores trimestrales. 
Como ya se ha señalado, inicialmente se dispone de una muestra de 64 empresas con observaciones trimestrales para todas las variables necesarias, durante un período de 6 años (2000-2005), es decir un total de 1.536 observaciones empresa-período de tiempo. No obstante se pierden 192 para poder calcular el primer período móvil (4Q2000), 64 para poder calcular la variación del Capital Circulante respecto del período inmediatamente anterior, y otras 64 para estimar el crecimiento que experimentan las ventas y el ratio CF/S. Por tanto, al disponer de 1.216 observaciones, se obtienen 1.216 valores calculados, que son comparados con los respectivos valores de mercado para cada empresa de la muestra, comprobando así la eficiencia que, en términos valorativos, tiene el modelo de valoración descrito en el presente trabajo.

\section{Estimación del crecimiento, las tasas de descuento y los múltiplos de valoración.}

Para poder realizar la proyección de los flujos de caja durante el primer intervalo y para obtener el importe estimado del conductor de valor (ventas y EBITDA) en el momento de cálculo del valor terminal, es necesario estimar las tasas de crecimiento de las ventas, del EBITDA y del ratio CF/S.

Según Damodaran (2002), existen varias manera de estimar el crecimiento esperado que una determinada variable experimenta a lo largo de un período de tiempo. Entre otras, dicho autor destaca la utilización de series temporales, la estimación mediante regresiones lineales y logarítmicas, o utilizando las estimaciones realizadas por analistas financieros. En este trabajo el crecimiento de la variable estudiada se estima en función en función de su variación respecto del período anterior. Si por ejemplo la variable en cuestión es el EBITDA, el crecimiento se estima mediante la siguiente ecuación:

$$
g_{e}=\ln \left(\frac{E_{i}}{E_{i-1}}\right)
$$

En el presente trabajo, se ha establecido una duración para el primer intervalo de 5 años, por lo que las tasas calculadas según el procedimiento anterior sirven para proyectar la evolución esperada de la variable durante esos 5 períodos.

Respecto a la tasa de descuento, es necesario en primer lugar estimar el coste de los recursos propios mediante la expresión del CAPM, para posteriormente calcular el coste medio ponderado de todas las fuentes de financiación (WACC) a partir también del coste de la deuda (datos obtenidos en Damodaran, 2006).

Los múltiplos de valoración para cada empresa se obtienen como media simple de los múltiplos observados en dicha empresa durante el horizonte de estudio. Se ha optado por esta opción, ya que según se observa en los valores presentados en el Anexo 1, existen fuertes variaciones para dichos valores entre las empresas de la muestra, imposibilitando la estimación de un único múltiplo representativo del sector analizado.

En futuros trabajos se pretende controlar dichas diferencias para poder extraer múltiplos representativos de grupos de empresas comparables, bien mediante la segmentación en grupos homogéneos de empresas que presenten múltiplos de valoración similares, bien mediante la segmentación en función de otra serie de variables anteriormente comentadas, como por ejemplo margen EBITDA sobre ventas, inversión en capital-ventas, etc. 


\section{1.- Eficiencia valorativa del modelo}

Para contrastar la eficiencia en términos valorativos del modelo se ha efectuado un análisis mediante dos regresiones lineales simples, donde la variable dependiente en ambas es el valor de mercado de la empresa (calculado como la suma de la capitalización bursátil más el valor contable de la deuda financiera neta). Como variables independientes actúan el valor calculado por aplicación del modelo cuando: i) se estima el valor terminal mediante el múltiplo de las ventas y, ii) mediante el múltiplo del EBITDA respectivamente.

El método anterior (regresión lineal) es el más utilizado en la investigación académica para contrastar si un determinado modelo de valoración explica de manera fiable el valor de mercado. Ejemplos de esto último son los trabajos de Charitou y Ketz (1991), Kaplan y Ruback (1995,1996), y Liu et al. (2002).

La expresión de la regresión es la siguiente:

$$
E V_{i, t}=\alpha+\beta \cdot X_{i, t}+\varepsilon
$$

Con $\mathrm{i}=1, \ldots 64 \mathrm{y} \mathrm{t}=1, \ldots 19$. Donde $\varepsilon$ representa el error cometido en la valoración, $\alpha$ y $\beta$ son los parámetros a estimar, y X representa el valor calculado según los dos métodos anteriormente descritos.

Para observar la correlación existente entre las variables de la ecuación anterior, se ha efectuado un test de correlación no paramétrica (Rho de Spearman), ya que ninguna de las variables se distribuye siguiendo una distribución normal (se ha obtenido un p-valor $<0.00$ en la prueba de Kolmogorov-Smirnov para las tres variables). Los resultados obtenidos muestran una correlación significativa (0,01 bilateral) entre el valor de mercado (EV) y el valor calculado mediante múltiplo de ventas-CV $(0,942)$ y entre valor de mercado y el valor calculado mediante múltiplo del EBITDA-CVE (0,887).

\section{Resultados de la regresión}

La Tabla 1 presenta los resultados más significativos de ambas regresiones:

\begin{tabular}{|c|c|c|c|c|}
\hline Regresión & Constante & $\boldsymbol{\beta}$ & $\mathbf{R}^{2}$ corregida & Durbin-Watson \\
\hline EV vs. CV & $\begin{array}{c}5762,343^{7} \\
(.000)\end{array}$ & $\begin{array}{c}0,615 \\
(.000)\end{array}$ & 0.667 & 1,182 \\
\hline EV vs. CVE & $\begin{array}{c}8083.628^{7} \\
(.000)\end{array}$ & $\begin{array}{c}0.470 \\
(.000)\end{array}$ & 0.549 & 0.953 \\
\hline
\end{tabular}

Tabla 1: Regresión valor de mercado vs. Valor calculado

Según los datos anteriores, puede deducirse que el modelo de valoración propuesto en este trabajo permite obtener resultados fiables en cuanto a eficiencia valorativa se refiere, mejorando los resultados obtenidos cuando el múltiplo utilizado para estimar el valor terminal de la inversión es el múltiplo EV/S. Dichos resultados, son consistentes con los obtenidos por autores como Charitou y Ketz (1991) y Kaplan y Ruback (1995,1996), mejorando incluso alguno de ellos.

Determinados autores proponen, con el objetivo de mejorar la eficiencia valorativa de un determinado modelo, escalar ambos miembros de la Ecuación 15 por alguna variable significativa de la muestra contrastada. Por ejemplo, Baker y Ruback (1999), Beatty et al. (1999), y Liu et al. (2002) dividen ambos miembros de la

\footnotetext{
${ }^{7}$ Millones de dólares americanos.
} 
expresión por el precio de la acción, concluyendo que actuando de esta manera permite obtener estimadores más precisos en la regresión. En este trabajo, se ha repetido el estudio anterior, dividiendo ambos miembros de la expresión entre el Activo Total de cada empresa, para cada período de estudio. La Tabla 2 presenta los resultados obtenidos:

\begin{tabular}{|c|c|c|c|c|}
\hline Regresión & Constante & $\boldsymbol{\beta}$ & $\mathbf{R}^{2}$ corregida & Durbin-Watson \\
\hline EV' vs. CV' & $\begin{array}{l}0.582 \\
(.000)\end{array}$ & $\begin{array}{l}0.639 \\
(.000)\end{array}$ & 0.531 & 0,991 \\
\hline EV' vs. CVE' & $\begin{array}{l}1,477 \\
(.000)\end{array}$ & $\begin{array}{l}0.196 \\
(.000)\end{array}$ & 0.182 & 0,475 \\
\hline
\end{tabular}

Tabla 2: Regresión valor de mercado vs. Valor calculado escalado por el Activo Total.

Puedo observarse que los resultados no mejoran, siendo en algún caso (cuando se estima el valor terminal con el múltiplo EV/E), significativamente peores.

No obstante, hay que ser extremadamente cautelosos en la interpretación de los datos, puesto que tal y como puedo observarse en las Tablas 1 y 2, los valores obtenidos para el estadístico de Durbin-Watson señalan la existencia de autocorrelación entre los términos de error obtenidos. Esto último puede ser debido a la metodología empleada en el cálculo de las variables contables que alimentan al modelo, obtenidas sobre la base de períodos móviles para evitar la estacionalidad de los datos. En futuros trabajos se intentará solucionar lo anterior utilizando otra tipo de metodologías, como por ejemplo datos de panel.

Otra posible causa de dicha autocorrelación es que en algunas empresas de la muestra el múltiplo medio presenta un coeficiente de variación superior a 0,25 (Anexo 1). Si se hubieran eliminado dichas empresas de la muestra los resultados mejorarían considerablemente.

Como conclusión, parece razonable afirmar que la combinación del modelo DCFM y múltiplos de valoración expresada en la Ecuación 13 aplicada sobre datos contables y valor de mercado de la empresa en el momento de la valoración, explica significativamente los valores de mercado de las empresas valoradas.

\section{6.- PRINCIPALES REFLEXIONES Y FUTURAS LÍNEAS DE INVESTIGACIÓN.}

Esta comunicación presenta una variante del modelo de descuento de flujos de caja para la valoración de empresas. El horizonte de valoración se divide en dos intervalos: en el primero de ellos se realiza una proyección detallada de los flujos de caja, basada en la utilización del ratio flujo de caja-ventas, su tasa de variación, y en la evolución de la cifra de ventas, durante $n$ períodos (cinco en este caso); en el segundo se estima un valor terminal para la inversión, mediante la aplicación de dos múltiplos de valoración relativa (múltiplo valor total-ventas, y valor total-EBITDA).

Los resultados obtenidos señalan la utilidad que en términos valorativos tiene la utilización de información contable y la combinación del modelo de descuento de flujos de caja con los métodos de valoración relativa. No obstante, tal y como se ha señalado en el apartado anterior, debido al grado de autocorrelación existente entre los residuos, los resultados deben ser interpretados con cautela. En futuros trabajos se pretende utilizar otras alternativas de estudio que permitan solucionar el problema anterior, para que dichos resultados sean más fiables, desde un punto de vista econométrico. 
En cuanto a las futuras líneas de investigación, señalar que pretende escalarse el modelo propuesto en el presente trabajo, desde un único ratio sintético, a cada uno de los ratios obtenidos al relacionar las distintas componentes de cálculo de los flujos de caja con las ventas. Se realizará un análisis estructural de composición y evolución histórica de los distintos ratios que relacionan componentes de cálculo de los FCFE con las ventas, para poder determinar la estructura de la empresa valorada. Estructura en términos de margen de EBITDA sobre ventas, porcentaje que representa la inversión en capital y la variación del circulante sobre el total de las ventas, etc. De esta manera se espera conseguir una predicción más fiable de la evolución de los flujos de caja durante el período de proyección, lo que redundaría en una mejora en los resultados de la valoración.

De la misma manera, puede adaptarse fácilmente a la valoración del neto patrimonial, si los flujos utilizados son los teóricamente disponibles para los accionistas. En determinados casos, cuando así sea posible y necesario, el modelo puede adaptarse también para recoger los distintos sectores de actividad de las empresas valoradas, donde cada uno de ellos será distinto al resto de segmentos, en cuanto a rentabilidad, crecimiento y diferencial de riesgo se refiere.

Finalmente, señalar también, que puede extenderse el modelo aquí discutido incorporando algún intervalo intermedio de valoración, anterior al valor terminal, para recoger etapas de transición entre intervalos. De esta manera, se consigue disminuir el porcentaje del valor de mercado explicado por el valor terminal.

\section{BIBLIOGRAFÍA}

(1) Alford, A. (1992). The effect of the set of comparable firms on the accuracy of the Price-earnings valuation method. Journal of Accounting Research 30, 94-108.

(2) Baker, M., Ruback, R. S. (1999). Estimating Industry Multiples. Working paper, Harvard University, Cambridge, MA.

(3) Beatty, R.P., Riffe, S.M., Thompson, R. (1999). The Method of Comparables and Tax Court Valuations of Private Firms: An Empirical Investigation. Accounting Horizons 13, 177-199.

(4) Beaver, W., Morse, D. (1978). What determines price-earnings ratios?. Financial Analysts Journal 34, 65-76.

(5) Beaver, W., Ryan, S. (2000). Biases and lags in book value and their effects on the ability of the book-to-market ratio to predict book rate of return on equity. Journal of Accounting Research 38, 127-148.

(6) Bhojraj, S., Lee, C.M.C. (2002). Who is my Peer? A Valuation-Based Approach to the Selection of Comparable Firms. Journal of Accounting Research 40, 407-439.

(7) Charitou, A., Ketz, E. (1991). An empirical examination of cash flow measures. Abacus 27 (1), 5164.

(8) Damodaran, A. (1994). Damodaran on Valuation. Wiley.

(9) Damodaran, A. (2001). The Dark Side of Valuation. Financial Times-Prentice Hall. 
(10) Damodaran, A. (2002). Investment Valuation (2a ed).Wiley.

(11) Damodaran, A. (2006). Damodaran Online-Web Page for Aswath Damodaran.

(www.pages.stern.nyu.edu/ adamodar/).

(12) Derrig, R.A., Orr, E.D. (2004). Equity Risk Premium: expectations great and small. North American Actuarial Journal 8 (1), 45-69.

(13) Dehning, B., Richardson, V.J., Stratopoulos, T. (2005). Information technology investments and firm value. Information and Management, 42, 989-1008.

(14) Eberhart, A.C. (2001). Comparable Firms and the Precision of Equity Valuations. Journal of Banking \& Finance 25, 1367-1400.

(15) Fama, E., French, K. (2000). Forecasting profitability and earnings. Journal of Business 73, 161175.

(16) Giner, B. (2001). La utilidad de la información contable desde la perspectiva del mercado: ¿evolución o revolución en la investigación?. Revista de Contabilidad 4 (7), 21-52.

(17) Giner, B., Reverte, C., Arce, M. (2002). El papel del análisis fundamental en la investigación del mercado de capitales: análisis crítico de su evolución. Revista Española de Financiación y Contabilidad 31 (114), 1111-1150.

(18) Gordon, M.J., Shapiro, E. (1956). Capital equipment analysis: The required rate of profit. Management Science 3, 102-110.

(19) Gordon, M.J. (1962). The investment, financing and valuation of the corporation. Richard D. Irwin.

(20) Graham, J.R., Harvey, C.R. (2001). The theory and practice of corporate finance: evidence from the field. Journal of Financial Economics, 60, 187-243.

(21) Kaplan, S.N., Ruback, R.S. (1995). The Valuation of Cash Flow Forecast: An Empirical Analysis. Journal of Finance 50, 1059-1093.

(22) Kaplan, S.N., Ruback, R.S. (1996). The Market Pricing of Cash Flow Forecasts: Discounted Cash Flow vs. the Method of Comparables. Journal of Applied Corporate Finance 8, 45-60.

(23) Kim, M., Ritter, J. (1999). Valuing IPOs. Journal of Financial Economics 53, 409-437.

(24) Koller, T., Goedhart, M., Wessels, D. (2005). Measuring and Managing the Value of Companies ( $4^{\mathrm{a}}$ ed). Wiley.

(25) Kothari, S.P. (2001). Capital Markets Research in Accounting. Journal of Accounting and Economics 31, 105-231.

(26) Kruschwitz, L., Löffler, A. (2006). Discounted cash flow: A theory of the valuation of firms. Wiley.

(27) Liu, J., Nissim, D., Thomas, J. (2002). Equity Valuation Using Multiples. Journal of Accounting Research 40, 135-172. 
(28) Molodovsky, N. (1953). A theory of price-earnings ratios. Financial Analysts Journal 9, 65-80.

(29) Nissim, D., Penman, S.H. (2001). Ratio Analysis and Equity Valuation: From Research to Practice. Review of Accounting Studies 6, 109-154.

(30) Ohlson, J.A. (1995). Earnings, book values and dividends in equity valuation. Contemporary Accounting Research 11, 661-687.

(31) Ohlson, J.A., Zhang, X.J. (1999). On the Theory of Forecast Horizon in Equity Valuation. Journal of Accounting Research 37, 437-449.

(32) Peasnell, K. (1982). Some formal connections between economic values and yields, and accounting numbers. Journal of Business Finance and Accounting 9, 361--381.

(33) Penman, S. (1996). The articulation of price-earnings rations and market-to-book ratios and the evaluation of growth. Journal of Accounting Research 34, 235-259.

(34) Penman, S. (1998a). A synthesis of equity valuation techniques and the terminal value calculation for the dividend discount model. Review of Accounting Studies 2, 303-323.

(35) Penman, S. (1998b). Combining earnings and book values in equity valuation. Contemporary Accounting Research 15, 291-324.

(36) Preinreich, G. (1938). Annual survey of income theory: the theory of depreciation. Econometrica 6, 219-241.

(37) Ryan, S. (1995). A model of accrual measurement with implications for the evolution of the bookto-market ratio. Journal of Accounting Research 29, 95-112.

(38) Tasker, S.C. (1998). Industry preferred multiples. Working paper. Cornell University, Ithaca, NY.

(39) Trahan. E.A., Gitman, L.J. (1995). Bridging the theory-practice gap in corporate finance: A survey of chief financial officers. The Quarterly Review of Economics and Finance, 35 (1), 73-87. 
ANEXO 1: Múltiplos de valoración.

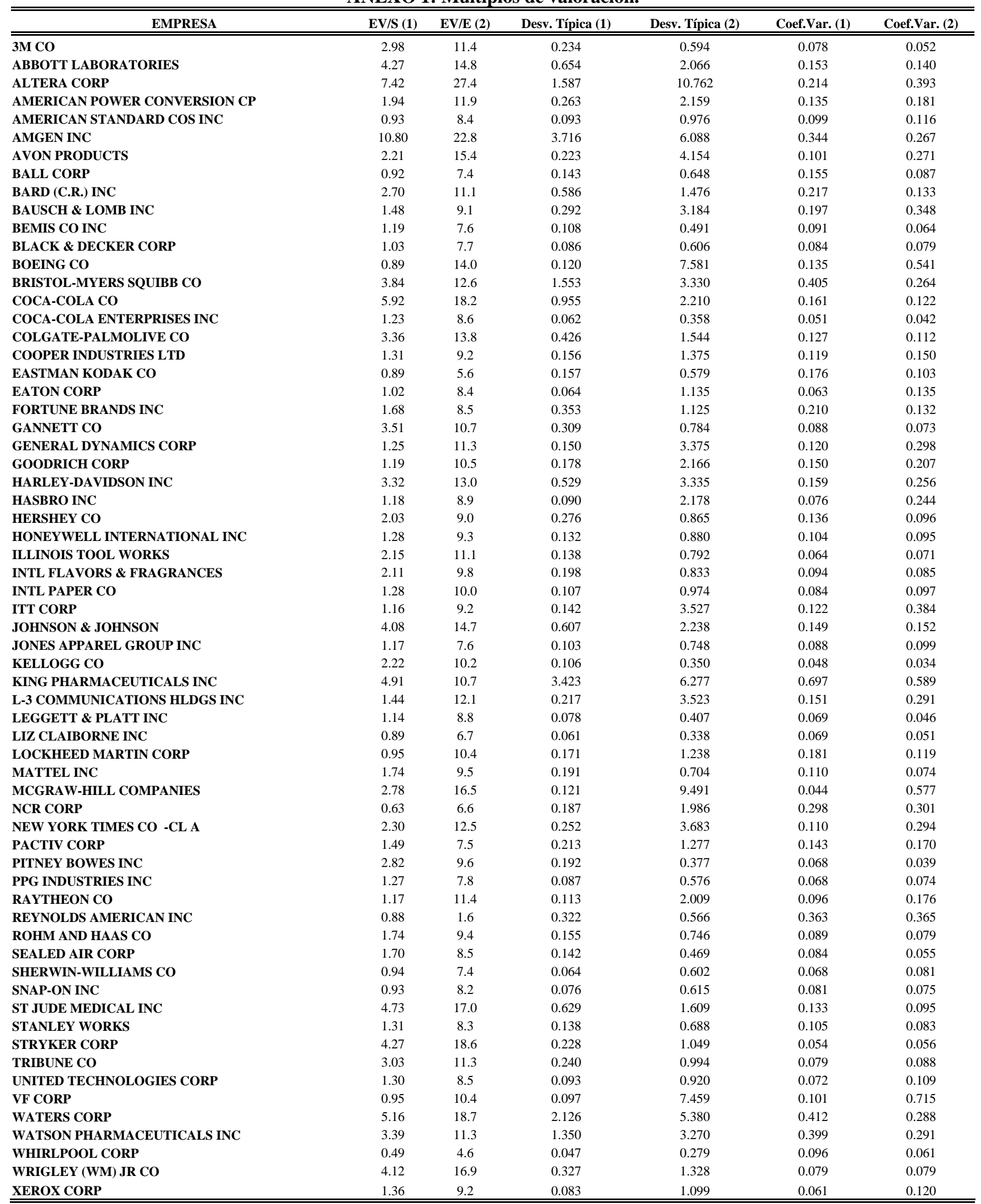

